\title{
CANCER DISEASE: INTEGRATIVE MODELLING APPROACHES
}

\author{
Helen M. Byrne, Markus R. Owen*, \\ Centre for Mathematical Medicine, \\ School of Mathematical Sciences, \\ University of Nottingham, \\ Nottingham NG7 2RD, \\ England,
}

\begin{abstract}
Cancer is a complex disease in which a variety of phenomena interact over a wide range of spatial and temporal scales. In this article a theoretical framework will be introduced that is capable of linking together such processes to produce a detailed model of vascular tumour growth. The model is formulated as a hybrid cellular automaton and contains submodels that describe subcellular, cellular and tissue level features. Model simulations will be presented to illustrate the effect that coupling between these different elements has on the tumour's evolution and its response to chemotherapy.
\end{abstract}

\section{INTRODUCTION}

Cancer is a complex and insidious disease in which controls designed to regulate growth and maintain homeostasis become disrupted. It is frequently initiated by genetic mutations that increase the net rate of cell division and lead to the formation of a small avascular lesion. Successful angiogenesis (i.e. the formation and ingrowth of a network of new blood vessels to the tumour) is needed before the extensive and rapid growth associated with vascularised tumours can occur. To confound matters, the processes involved in tumour growth are interlinked and act over a wide spectrum of spatial and temporal scales: the spatial scales of interest range from the subcellular level, to the cellular and macroscopic levels while the timescales may vary from seconds (or less) for signal transduction pathways to months for tumour doubling times.

The advent of increasingly sophisticated technology means that it is now possible to collect experimental data associated with the spatial and temporal scales of interest. This is creating a demand for new theoretical models that have the capacity to integrate such data in a meaningful manner and are

*The authors gratefully acknowledge financial support provided by the EPSRC under grants GR/5090067, GR/S72023/01 and AF/00067.

${ }^{\dagger}$ The third author is currently based at the Bioinformatics Unit, Department of Computer Science, University College London, Gower Street, London WC1E 6BT, England.

\author{
Tomas Alarcon ${ }^{\dagger}$ and Philip K. Maini, \\ Centre for Mathematical Biology, \\ Mathematical Institute, \\ 24-29 St Giles', \\ Oxford OX1 3LB, \\ England.
}

able to address the fundamental problem of how phenomena at different spatial scales are coupled.

In this paper we review our recent progress in developing a mathematical model for studying vascular tumour growth that is capable of integrating phenomena that act on different scales [1]. Our theoretical framework extends earlier work by Gatenby and coworkers [2] and links submodels which describe processes operating on different spatial scales. In section 2 we introduce our hybrid cellular automaton, before presenting numerical results in section 3 . The simulations illustrate how the coupling between the submodels influences the tumour's evolution and its response to chemotherapy. We conclude in section 4 with a summary of our results and a discussion of possible directions for future research.

\section{MODEL FORMULATION}

In this section we introduce our multiscale model of vascular tumour growth. It accounts for a variety of inter-related phenomena that operate on vastly different space and time scales. We consider a vasculature composed of a regular hexagonal network embedded in a two-dimensional $\mathrm{NxN}$ lattice composed of normal cells, cancer cells and empty space. Progress through the cell cycle and the production of proteins such as vascular endothelial growth factor (VEGF) that stimulate angiogenesis are incorporated at the subcellular level using ODE models. Cell-cell communication and competition for resources are included at the cellular level through rules that define our cellular automaton. At the tissue scale, reactiondiffusion equations model the diffusion, production and uptake of oxygen and VEGF: the vessels are regarded as sources (sinks) of oxygen (VEGF) and the cells as sinks (sources) of oxygen (VEGF). Blood flow and vascular adaptation are also included at the tissue scale (see Figure 1). We impose a pressure drop across the vasculature, assuming that blood flows into the idealised "tissue" through a single inlet vessel and drains out through a single outlet vessel. We use the Poiseuille approximation and compute the flow rates through, and pressure drops across, each vessel using Kirchoff's laws. 
The vessel radii are updated using a structural adaptation law similar to that proposed by Pries et al. [3] (for details, see [1]).

Thus the model is formulated as a hybrid cellular automata, with different submodels describing behaviour at the subcellular, cellular and macroscopic (or vascular) levels (see Figure 1). Coupling between the different submodels is achieved in several ways. For example, local oxygen levels which are determined at the macroscale influence both progress through the cell cycle and VEGF production at the subcellular level. Conversely, the intracellular production of VEGF modulates vascular adaptation at the macroscale and this, in turn, controls oxygen delivery to the tissue. We stress that the submodels we use simply illustrate how such a multiscale model can be assembled: the framework we present is general, with considerable scope for incorporating more realistic (i.e. complex) submodels. This raises the important issue of how the level of detail incorporated at each spatial scale influences the system's behaviour: this will form the basis of future research.

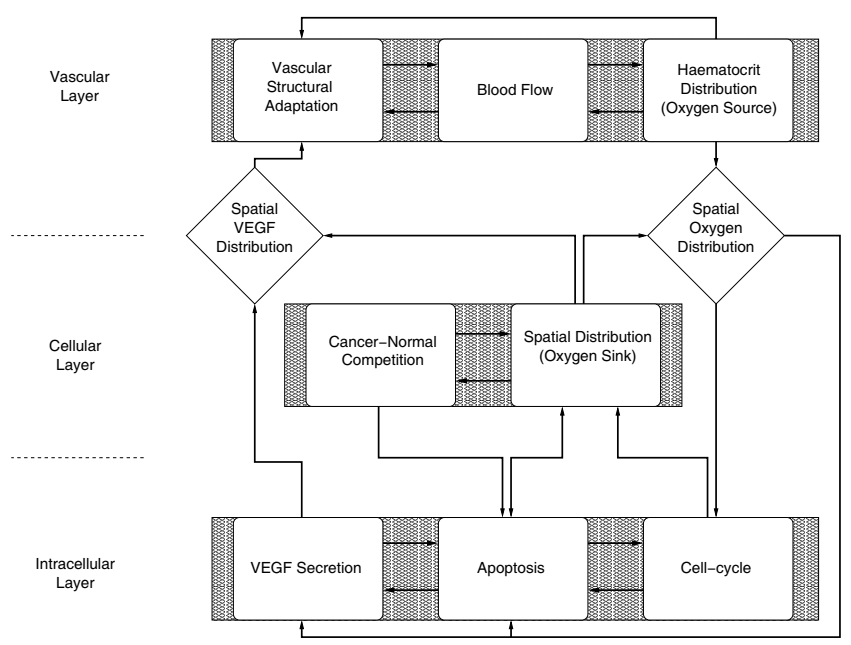

Fig. 1. Schematic showing the structure of our hybrid cellular automaton model. Reproduced with permission from [1].

\section{NUMERICAL RESULTS}

\subsection{Vascular adaptation influences tumour growth}

In Figure 2 we present simulations that illustrate the importance of accurately modelling blood flow through the tissue. The upper panels correspond to a case for which the vessels undergo structural adaptation and, hence, oxygen is distributed nonuniformly across the tissue. The lower panels show how the system evolves when oxygen is distributed uniformly throughout the vessels (i.e. blood flow is identical in all branches of the vasculature). We see that spatial heterogeneity has a significant effect on the tumour's dynamics and, in this case, actually reduces the tumour burden. We note also that if the oxygen distribution is heterogeneous then the tumour has "finger-like" protrusions similar to those observed in invasive cancers. This structure arises here simply because of the spatial heterogeneity in the nutrient distribution. Indeed, closer inspection reveals that several parts of the tumour have almost "broken away". While this cannot actually happen in the current model because cell motion is neglected, we speculate that by allowing cell movement towards nutrientrich regions, this may act as a mechanism for metastasis.

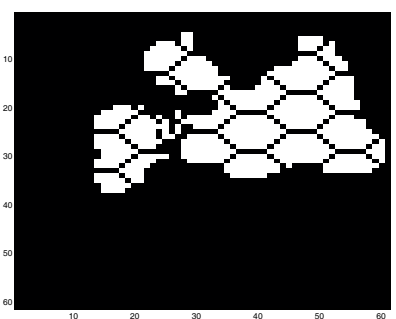

(a)

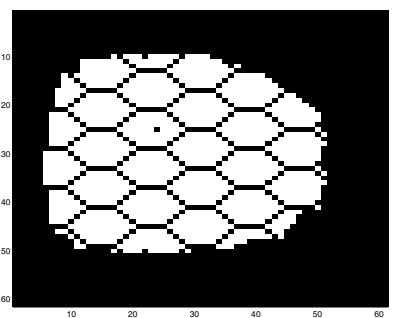

(c)

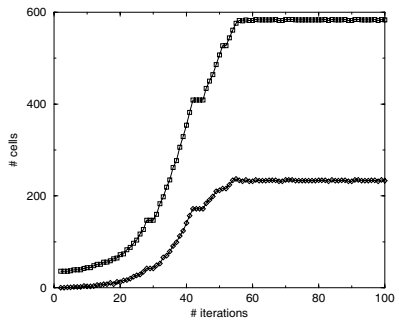

(b)

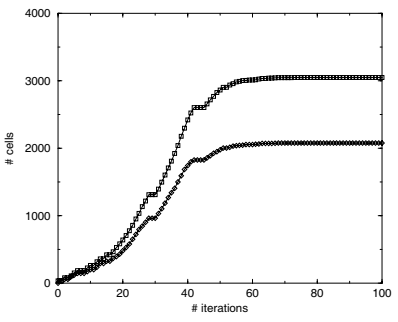

(d)
Fig. 2. Series of images showing the spatial distribution of cells for growth in inhomogeneous (panel a), and homogeneous environments (panels c). In panels (a) and (c) cancer cells occupy white spaces and vessels occupy a hexagonal arrray denoted by black spaces. The other black spaces denote "empty spaces". Panels (b) and (d) show the time evolution of the number of (cancer) cells for the heterogeneous and homogeneous cases, respectively: squares denote the total number of cancer cells (proliferating + quiescent); diamonds denote quiescent cells. Reproduced with permission from [1].

\subsection{Impact of VEGF on the tumour's growth dynamics}

The simulation presented in Figures 3 to 5 shows how coupling intracellular and macroscale phenomena can influence the dynamics of both the vasculature and the tumour. In contrast to the results depicted in Figure 2, where vessel adaptation was independent of VEGF, in Figures 3 to 5 it is regulated by local VEGF levels. Figures 3 and 4 show how the tumour's spatial composition evolves while figure 5 summarises its dynamics. Since there is a single inlet (outlet) to the vasculature located in the bottom left (top right) hand corner of the tissue, the incoming blood flow and haematocrit become diluted as they pass through the hexagonal lattice. This creates a het- 
erogeneous oxygen distribution across the domain, with oxygen levels being highest near the inlet and outlet. Over time, the tumour cells proliferate and spread through the tissue towards oxygen-rich regions. As they increase in number, their demand for oxygen outstrips that available from the vasculature, and quiescent regions form. These cells produce VEGF which diffuses through the tissue (see Figures 3 and 4), stimulating vessel adaptation and biasing blood flow towards low oxygen regions. If the VEGF stimulus is weak then the vasculature does not adapt quickly enough and the quiescent cells die (this is what happens at early times in figure 5). VEGF levels also decline and blood flow to the remaining tumour cells rises, enabling them to increase in number until the demand for oxygen once again exceeds that being supplied, and so the cycle repeats, with pronounced oscillations in the number of quiescent cells (see Figure 5). In order to highlight the key role played by VEGF in creating these oscillations, also presented in Figure 5 are the results of a simulation which was identical in all respects except that vascular adaptation was independent of VEGF (as per Figure 2). In both cases, the tumours grow to similar sizes. However, when vascular adaptation is independent of VEGF the evolution is monotonic, the oscillations in the cell populations disappear and the number of quiescent cells is consistently much lower. These results show how coupling between the different spatial scales can effect not only the tumour's growth dynamics but also the proportion of proliferating and quiescent cells that it contains.
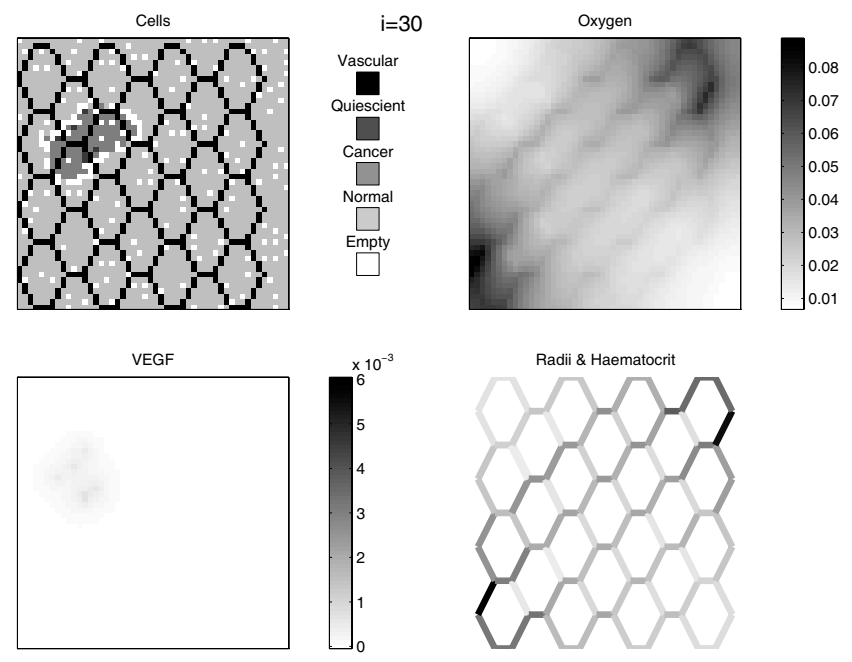

Fig. 3. Series of plots showing how a small tumour introduced into a vascular tissue at $t=0$ has evolved at $t=30$ (dimensionless time unit). While the oxygen and vessel profiles remain unchanged from their initial configurations, the tumour has increased in size and now contains quiescent cells which produce trace amounts of VEGF. Reproduced with permission from [4].
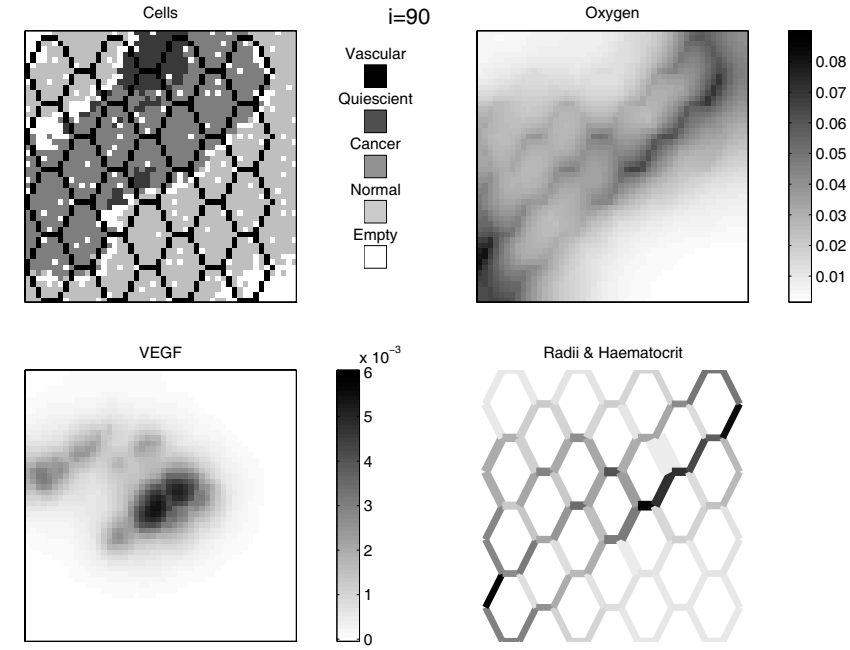

Fig. 4. Series of plots showing how the simulation presented in figure 3 has developed at $t=90$. The tumour continues to penetrate the tissue region. There are now enough quiescent cells to elicit an angiogenic response. As a result, the vasculature has been remodelled, with blood flow and oxygen supply (haematocrit) being directed primarily towards the tumour mass. Reproduced with permission from [4].

\subsection{Response to chemotherapy}

We now investigate how the system's dynamics change when a chemotherapeutic drug is introduced. We assume that the drug is continuously administered to the vessels and, hence, that its concentration at the inlet vessel is constant. We calculate the drug concentration within and outside the vessels in a manner similar to that used to determine the oxygen distributions. In particular, once the drug leaves the vessels, it diffuses through the tissue and must be taken up by the normal and healthy cells before it can act. For simplicity, we assume that the drug works in the following manner. When a cell attempts to divide, if the local drug concentration exceeds a threshold value then the cell fails to divide and is itself killed.

Repeated simulations suggest that, when the drug is used, three qualitatively different types of behaviour emerge: the drug is ineffective and the tumour continues to colonise the tissue, the tumour is reduced in size, or it is completely eliminated. Figure 6 summarises the tumour dynamics associated with the different possible outcomes. The simulations were obtained by varying a parameter $\left(h_{\theta}\right)$ that measures the rate at which the drug extravasates. Since increasing $h_{\theta}$ corresponds to tumour regions with more permeable vessels, our results suggest that a drug of this type is likely to have greater specificity in tumour regions containing immature and leaky vessels. Alternatively, if we interpret decreasing $h_{\theta}$ as using drugs with larger molecular weights then our results suggest that smaller drugs will be delivered more readily and, hence, evoke a stronger cell kill than larger, heavier drugs. 

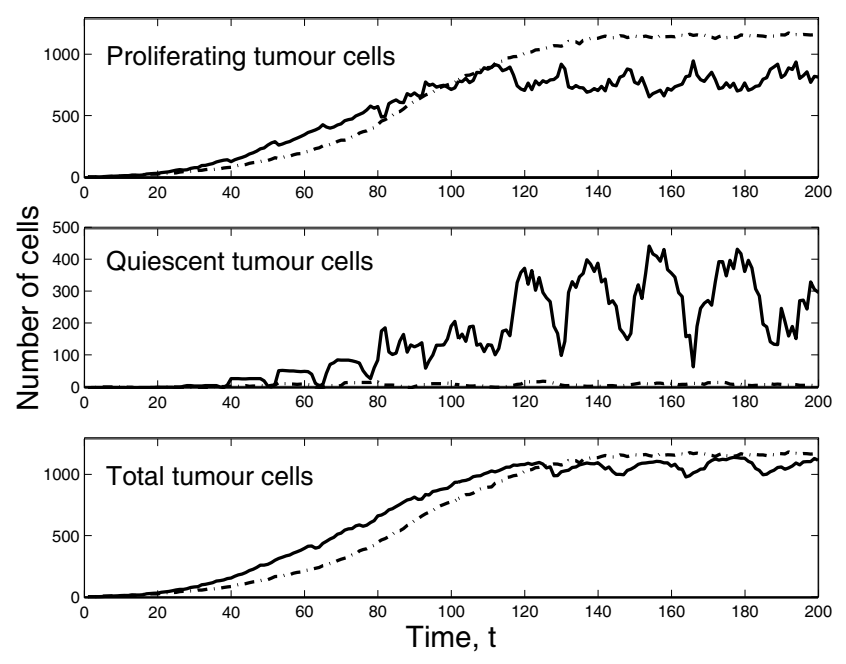

Fig. 5. Series of curves showing how, for the simulation in figures 3 and 4 the numbers of proliferating (upper panel), quiescent tumour cells (middle panel) and total number of tumour cells (lower panel) change over time. While the number of proliferating cells increases steadily, the number of quiescent cells undergoes oscillations of increasing amplitude until $t \approx 120$. Thereafter, the tumour is sufficiently large that the quiescent cells are never eliminated: quiescent cells that die are replaced by proliferating cells that become quiescent. The dot-dashed lines show the evolution of a tumour which is identical except that its vasculature is not regulated by VEGF. While both tumours reach similar equilibrium sizes, when vascular adaptation is independent of VEGF the oscillations in the cell populations disappear and the number of quiescent cells is much lower. Reproduced with permission from [4].

\section{DISCUSSION}

We have presented a hybrid cellular automaton model of vascular tumour growth and shown how it may be used to study the manner in which interactions between subcellular, cellular and macroscale phenomena affect the tumour's growth dynamics and its response to chemotherapy. We stress that the submodels we have used to describe the different processes are highly idealised and chosen simply to illustrate the potential value of such a multiscale model as a predictive tool to test experimental hypotheses and to integrate different types of experimental data. There is considerable scope for incorporating more realistic submodels and specialising the system to describe specific tumour types. For example, we are currently engaged in a large interdisciplinary project which aims to build a virtual model of the early stages of colorectal cancer (details at: http: / /www. integrativebiology.ox.ac.uk ).

Key challenges raised by our simulations that lie at the heart of such integrative modelling concern the level of detail incorporated at each spatial scale, the mathematical approaches used and model validation. For example, in this
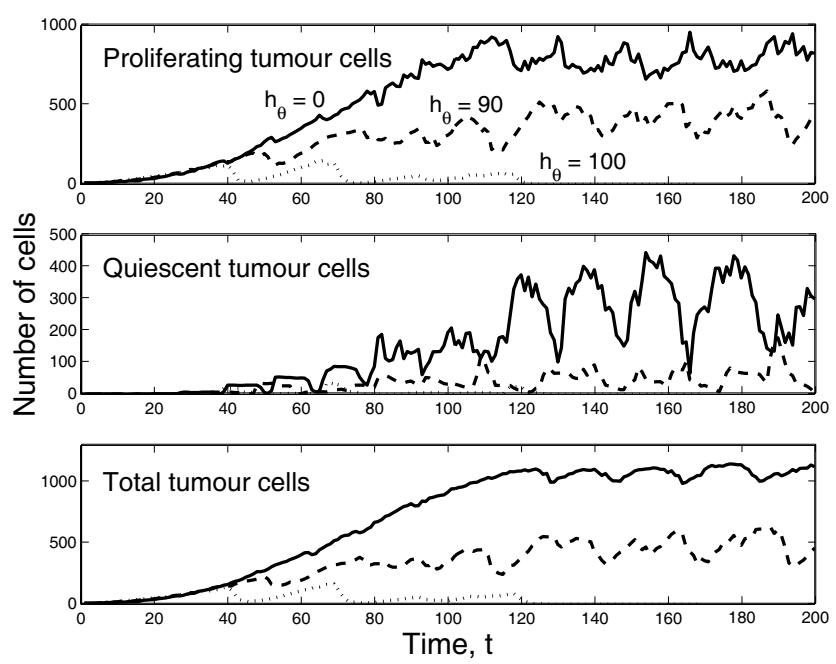

Fig. 6. Series of curves showing how the tumour's overall dynamics change when it is treated with two blood-borne chemotherapeutic agents that differ only in their extravasation rates. For each simulation, we plot the numbers of proliferating and quiescent tumour cells and the total number of tumour cells evolve over time. Key: $h_{\theta}=0$ (control, drug-free case, as per figure 5), solid line; $h_{\theta}=90$ (moderate drug), dashed line; $h_{\theta}=100$ (highly permeable and effective drug), dotted line. Reproduced with permission from [4].

article we chose to use a combination of differential equations and cellular automata to construct our virtual tumour. It remains an open question whether the predicted behaviour would change if we replaced our (subcellular) ODE models with Boolean networks and/or the cellular automata with agent-based models.

\section{REFERENCES}

[1] T. Alarcón, H.M. Byrne, and P.K. Maini, "A muliple scale model for tumour growth," SIAM J. Multiscale Mod. \& Sim., vol. 3, pp. 440-475, 2005.

[2] A.A. Patel, E.T. Gawlinsky, S.K. Lemieux, and R.A. Gatenby, "Cellular automaton model of early tumour growth and invasion: the effects of native tissue vascularity and increased anaerobic tumour metabolism," $J$. Theor. Biol., vol. 213, pp. 315-331, 2001.

[3] A.R. Pries, T.W. Secomb, and P. Gaehtgens, "Structural adaptation and stability of microvascular networks: theory and simulations," Am. J. Physiol., vol. 275, pp. H349H360, 1998.

[4] H.M. Byrne, M.R. Owen, T. Alarcón, J. Murphy, and P.K. Maini (in press), "Modelling the response of vascular tumours to chemotherapy: a multiscale approach," Math. Mod. Meth. Appl. Sci., 2005. 